\title{
Analysis of Characteristics of Chinese Patent Medicine Containing Radix Rehmanniae Preparata
}

\author{
Mengfan Peng ${ }^{1, a}$, Baosong Liu ${ }^{1, \mathrm{~b}}$, Ming Bai ${ }^{1, \mathrm{c}}$ and Mingsan Miao ${ }^{1, \mathrm{~d}, *}$ \\ ${ }^{1}$ School of Pharmacy, Henan University of Chinese Medicine, Zhengzhou, Henan, China \\ aliubaosong647@163.com, ${ }^{b} 1639975054 @ q q . c o m,{ }^{c} 948235483 @ q q . c o m,{ }^{d}$ miaomingsann@163.com \\ *corresponding author
}

Keywords: Radix Rehmanniae Preparata, Chinese Patent Medicine, Application and Analysis

\begin{abstract}
Radix Rehmanniae Preparata tastes sweet, tepor-natured, meridian tropism kidney and liver. It is the medicine for nourishing the liver and kidney, not only nourish the blood and nourish yin, but also nourish the essence and fill the marrow. In this paper, the authors summarized the Chinese patent medicines containing dandelion produced in the 2015 edition Chinese Pharmacopoeia, and analyzed its application forms, compositions, contraindications, indications. Finally, the relevant literature of CNKI was retrieved, and its clinical application was discussed and the correlation analysis was carried out.
\end{abstract}

\section{Introduction}

The Radices Rehmanniae, a medicinal plant which belongs to Scrophulariaceae is one of the Four Famous Huai Medicine and it has a long history [1]. From the Zhou Dynasty began, the Four Famous Huai Medicine was classified as a tribute. Tang and Song Dynasties, the Four Famous Huai Medicine has long been renowned, it was introduced to Europe and Asia through the silk road. In the Ming Dynasty, Zheng He brought the drugs into Southeast Asia, the Middle East and Africa. In modern times, with the Four Famous Huai Medicine is known as "Chinese Medicine" [2]. Radix Rehmanniae Preparata is composed of wine, amomum fruit, tangerine peel, radices rehmanniae, after repeated steam drying and processing. It tastes sweet, tepor-natured, meridian tropism kidney and liver. It is often used in the treatment of irregular menstruation, liver and kidney yin deficiency, dizziness, tinnitus and other symptoms [3]. Radix Rehmanniae Preparata contains many chemical constituents, such as iridoid, ajugol, rehmannioside, saccharide and amino acids. Modern pharmacology shows that Radix Rehmanniae Preparata has the effects of strengthening the heart, diuresis, anti inflammation, liver protection, antitumor and hypoglycemic effect [4]. Chinese patent medicine is processed and produced under the guidance of the basic theory of Chinese medicine. It contains active ingredients in various medicinal herbs and can treat complex diseases from different channels, different functions, targets and levels [5]. In order to provide reference for the development and utilization of Radix Rehmanniae Preparata and its Chinese patent medicines, the form, single herb number, functions indicated and application situation of Radix Rehmanniae Preparata Chinese patent medicine were summarized in this article. Then, the literature reports of the Chinese patent medicine containing Radix Rehmanniae Preparata were reviewed, and the application in clinical practice was summarized and narrated.

\section{The circumstances of Radix Rehmanniae Preparata Chinese patent medicines contained in the Chinese Pharmacopoeia 2015 edition[6]}

In the 2015 edition Chinese Pharmacopoeia, 140 kinds of Chinese patent medicine containing Radix Rehmanniae Preparata were recorded. In the Chinese patent medicines, the least is composed of 4 kinds of Chinese medicinal compositions (Siwuheji, Siwu granules, Fumingpian), the largest is composed of 58 
original herbs or their extracts (Zaizao pills). Zaizao pills and Qizhixiangfu pills were prepared with Radix Rehmanniae Preparata only 20g, but Shugukang capsule has up to $1656 \mathrm{~g}$. This paper divides it into 5 sections, which are: 1-5 flavors, 6 kinds; 6-10 flavors, 52 kinds; 11-15 flavors, 37 kinds; 16-20 flavors, 22 kinds; $>20$ flavor, 23 kinds. Among them, the pills, tablets, capsules and granules are mainly accounted for $88.6 \%$ of all Chinese patent medicines. The paste, mixture and oral liquid occupy $8.6 \%$ of all Chinese patent medicines, and other forms of dosage, such as syrup, occupy $2.8 \%$ of all Chinese patent medicines.

\section{The application form of Radix Rehmanniae Preparata Chinese patent medicines contained in the Chinese Pharmacopoeia 2015 edition}

140 kinds of Chinese patent medicine are containing Radix Rehmanniae Preparata, they were applied in 4 forms: water frying, smash, alcohol extraction and deep frying. Water frying and smash are the most important forms of Radix Rehmanniae Preparata in Chinese patent medicines, 74 and 60 respectively, accounting for $52.8 \%$ and $42.9 \%$ respectively. There are 5 kinds of Radix Rehmanniae Preparata are used as medicine in the form of alcohol extract, only 1 kind is used as medicine in the form of fried form. It is obvious that water frying and smash are the most common forms of Radix Rehmanniae Preparata. The method of alcohol extraction can change the solubility of Chinese medicine, remove some water-soluble impurities, and give better effect. Zihuashaoshang ointment used in sesame oil will Shudihuang fried to internal brown, extracting effective ingredients of Radix Rehmanniae Preparata by using hot oil, the active ingredient is dissolved in oil.

\section{Different classification and analysis of Chinese patent medicine containing Radix Rehmanniae Preparata}

140 kinds of Chinese patent medicines were classified according to function of Radix Rehmanniae Preparata. By summarizing, we can roughly classify the related Radix Rehmanniae Preparata in preparation of Chinese patent medicine into 4 broad categories. 1. Promoting blood circulation to restore menstrual flow: Wujibaifeng pill and Bazhenyimu pill. 2. Warmly invigorating kidney yang: Yougui pill and Shenbao mixture. 3. Nourishing yin and tonifying kidney: Zhibaidihuang pill and Guishaodihuang pill. 4. Dispel the wind and vein relaxing: Wangbipian and Zaizhao pill. These four groups contain 51 kinds of Chinese patent medicines, 35 kinds, 39 kinds and 15 kinds, and the percentages are $36.4 \%, 25 \%$, $27.9 \%$ and $10.7 \%$ respectively.

\section{The application and analysis of Chinese patent medicine containing Rehmannia glutinosa}

Radix Rehmanniae Preparata has the effects of nourishing blood and Yin, tonifying essence and filling marrow, mainly used for the treatment of blood deficiency, liver and kidney deficiency. When applied, it is used in combination with other medicines.

It represents six kinds of Chinese medicine rehmannia pills, Siwu mixture and so on.

\section{A. Nourishing blood yin}

Radix Rehmanniae Preparata is nourishing yin and blood products, taste sweet and sour. Neijing has recorded that if the semen is not enough, it should be filled with Radix Rehmanniae Preparata. Guishao Dihuang pills is made up by Radix rehmanniae, Angelica, Radix Paeoniae Alba, Cornus officinalis, cortex moutan and so on, it has the functions of clearing deficient heat, nourishing the liver, kidney and Yin blood. Li Qiaoli used Guishao Dihuang Pill relative prescription in treatment of liver blood deficiency in patients with recurrent oral ulcer. The results showed that the clinical efficacy of the patients was significantly improved, the level of inflammation in the body was reduced effectively, and the immune function of the body was improved, which had the clinical popularization and application 
value [7]. Weixuening mixture is made up by Radix rehmanniae, Polygonum cuspidatum, Radix Paeoniae Alba, Caulis Spatholobi, Radix Pseudostellariae, it has the functions of enriching blood and promoting fluid production, nourishing the kidney and the liver, clearing heat and reducing fever. Sun Jun studied the effects of different doses of Nourishing Yin blood pill and Siwu Decoction on blood deficiency mice, and found that they both have blood enriching effectively [8].

\section{B. Tonifying essence and filling marrow}

"Compendium of Materia Medica" records Radix Rehmanniae Preparata can fill the bone marrow, muscle, health blood, nourishing organ. Qiangshenpian is composed of pilose antler, Chinese yam, dogwood, prepared rehmannia root, medlar, Salvia miltiorrhiza, psoralen, cortex moutan and so on. Compatibility of Radix Rehmanniae Preparata with Chinese yam, it can increase the effect of Nourishing Yin, tonifying kidney, fixing essence, nourishing spleen and kidney at the same time. Zheng [9] and other studies have found that the strong kidney tablet can increase the bone mineral density and bone mineral content of the femur in osteoporotic rats, and have a antagonism effect on osteoporosis in rats. Shenbao syrup is composed of Fructus Psoraleae, Poria, Atractylodes, angelica, raspberry, rehmannia root, wolfberry fruit, Chinese yam, epimedium, licorice and so on. Compatibility of Radix Rehmanniae Preparata with Chinese wolfberry. It can increase the effect of Nourishing spleen and kidney Guse. Li [10] observed the therapeutic effect of Radix Rehmanniae Preparata and Nerve growth factor on spinal cord injury. The result showed that it had better therapeutic effect and was worthy of clinical application.

\section{Expelling wind and dredging collaterals}

Wangbipian comprises prepared rhizome of rehmannia, rehmannia, monkshood, cassia twig, epimedium etc., with tonifying the liver and kidney, strong bones, and the effect of dispelling wind and dredging collaterals. Commonly used in clinical liver and kidney deficiency, stasis caused by rheumatism muscle and joint pain, chills, fatigue and other symptoms. Wu Ting [11] found that Wangbi tablet combined with massage have a good clinical efficacy for treatment of knee osteoarthritis (KOA). Kangguzengsheng pill comprises Radix Rehmanniae Preparata, Rhizoma cibotii, epimedium, radish seed, achyranthes etc, with invigorating kidney, strong bones and muscles, promoting blood circulation and relieving pain, commonly used in clinical osteoarthritis of liver and kidney deficiency and blood stasis due to joint swelling, numbness, pain, activity limitation and other symptoms. Huang [12] studied the efficacy of anti proliferative capsule and sodium hyaluronate in the treatment of knee osteoarthritis, the results show that it can effectively relieve knee pain and promote the recovery of knee function.

\section{Outlook}

The prepared rehmannia root, also known as the Radix Rehmanniae Preparata, was first published in ShenNongBenCaoJing. It was juxtaposed with the top grade and was one of the commonly used traditional Chinese medicines [13]. In the 2015 edition of the Chinese Pharmacopoeia, 140 Chinese patent medicines containing Radix Rehmanniae Preparata were recorded, and the dosage form is rich. Rehmannia six, Qijudi huangwan, zhibaidi huangwan have better visibility, the clinical application of Radix Rehmanniae is widely used in clinical practice. Chinese patent medicine is under the guidance of the theory of traditional Chinese medicine, the Chinese medicinal material is made into the finished product of a certain preparation according to the prescribed prescription, and has the advantages of various varieties, complete dosage forms, convenient taking and the like [14]. Therefore, the market of Chinese patent medicine containing Radix Rehmanniae is broad and has a better prospect of development.

Rehmannia glutinosa is one of the four heavenly kings in traditional Chinese medicine, and it is recorded in Drug Huayi: Radix Rehmanniae Preparata, steam with wine, taste changed from bitter to sweet, nature changed from cool to warm, devoted to the liver and enriching the blood. Rehmannia glutinosa plays a strong role on enriching the blood, is recognized as the blood top grade [15]. Although 
the honey prepared Rehmannia (blood nourishing), Siwu Decoction (tea), Huai Rehmannia (instant use) is widely producted, its products are of simple processing, lacking of Rehmannia glutinosa related products of nourishing the blood as the main efficacy [16]. The study on the deep processing of the blood is enriching products of the Radix Rehmanniae Preparata accords with the functional characteristics of itself, and is also the embodiment of the curative effect of the genuine medicinal herbs.

Treatment based on syndrome differentiation is the characteristics of traditional Chinese medicine, Chinese patent medicines are mainly treated with dialectical drugs in the treatment of various diseases, reflecting the concept of different diseases with same treatment and treating the same disease with different methods. Only on the basis of syndrome differentiation and drug treatment can the characteristics and advantages of Chinese patent medicine be fully developed. In the future, we should focus on the compatibility of Radix Rehmanniae Preparata in Chinese patent medicines, clarify its role in the prescription and explain it in the instructions. It is also one of the important research directions of the future to continue the deep processing research, optimize the processing technology and give full play to the advantages of authentic herbs.

\section{Acknowledgment}

This research was financially supported by the National International Cooperation Base (NSIL2016-65), Henan provincial campus cooperation rewards and subsidies (Finance and Education (2016) No. 38), Henan Production, Education and Research Project (142107000039).

\section{References}

[1] Li Jinhua, Li Jun, Wang Junming, Cui Ying. Research progress on chemical constituents and pharmacological activities of adhesive rehmannia leaf [J]. Journal of Gerontology. 2016, 36 (19): 49264927.

[2] Liu Zhiyong. "Four Famous Huai Medicine" said Rehmannia glutinosa [J]. Chinese Medicine Health Regimen. 2016, (12): 31-32.

[3] Zhang Fuxing, Qiu Shengliang, Zhu Yutian, Research progress in extraction technology and pharmacological action of Polysaccharides from Radix Rehmanniae Preparata [J]. Zhejiang Journal of Traditional Chinese Medicinel. 2013, 23 (02): 153-154.

[4] Wei Guifang, Liu Xueping, He Xirui. Pharmacological and clinical application of Rehmannia glutinosa [J]. Shanxi Chinese Medicine. 2013, 34 (08): 1073+1096.

[5] Deng Shuqiong. Effect of Chinese patent prescription review on the rationality of clinical medication [J]. The Contemporary Medical Journal. 2017, 15 (10): 5-6.

[6] National Pharmacopoeia Committee. Pharmacopoeia of People's Republic of China. Beijing: China Medical Science and Technology Press, 2015.

[7] Li Qiaoli. Guishao Dihuang Decoction Combined with conventional therapy in the treatment of liver blood deficiency type of recurrent oral ulcer and effect on immune function [J]. Modern Journal of Integrated Traditional and Western Medicine. 2017, 26 (15): 1668-1670.

[8] Sun Jun, study on the effect of Nourishing Yin, nourishing blood pill and Siwu Decoction on blood stasis in mice with blood deficiency [J]. Journal of Changchun College of Traditional Chinese Medicine. 2005, (04): 38-39.

[9] Zheng Jiyu, Wu Yongjun, Wang Shijie, Qin Peihong, in Yaohong, Zang Linghe, Xu Feng. Jiangshen tablets effect on glucocorticoid induced osteoporosis in rats [J]. Pharmacology and Clinic of Chinese Materia Medica. 2015, 31 (01): 180-182. 
[10] Li Shuiqin, Gao Xiang, Li Xiaoling. Observation of curative effect of warming kidney, strengthening yang, tonifying essence, filling marrow and nerve growth factor in treating spinal cord injury [J]. Chinese Journal of Modern Chinese Medicine and Western Medicine. 2016, 25 (25): 2751-2753+2757.

[11] Wu Tinghuan, Zhou Hui, Chen Xingkai. Observation of massage combined with Wangbi pian in the treatment of osteoarthritis of the knee [J]. Chinese Medical Journal. 2017, 25 (01): 38-41.

[12] Huang Weijun, Pan Yali, Li Zhimin, Zhang Zeyu. United glass Zengsheng capsule oral sodium intraarticular injection in the treatment of knee osteoarthritis [J]. Traditional Chinese Medicine. 2017, 29 (04): 67-68.

[13] Guo Huanfang, Ma Li, Zhang Chao, Yan Yaping, Zhang Chunyan. 3 clinical cases of new clinical cases of addition and subtraction of Astragalus six flavored Rehmannia soup [J]. Hunan Journal of Traditional Chinese Medicine. 2015, 31 (09): 104-105.

[14] Li Xuebai. Discussion on incompatibility between Chinese patent medicine and Western medicine in emergency use of Chinese medicine [J]. Chinese Medicine. 2017, 36 (10): 89-90.

[15] Li Xian, Wang Na, Wei Zhi long. Study on the processing mechanism of the effect of Radix Rehmanniae Preparata on blood stasis [J]. Chinese Journal of Experimental Chinese Medicine. 2017, 23 (04): 29-33.

[16] Zhang Cai, Zhang Ping, Si Wenqi, Zhao Yan, Shi Lei. Extraction process of Rehmannia glutinosa D from Rehmannia glutinosa and comparison of the contents of different processed products [J]. Shandong Agricultural Sciences. 2016, 48 (05): 127-130. 